\title{
Changes in the self-rated well-being of people who move from congregated settings to personalized arrangements and group home placements
}

Journal of Intellectual Disabilities 2018, Vol. 22(I) 49-60

(C) The Author(s) 2016 Reprints and permission: sagepub.co.uk/journalsPermissions.nav DOI: $10.1177 / 1744629516674086$ journals.sagepub.com/home/jid

\section{Roy McConkey}

Ulster University, UK

Fiona Keogh

Genio Ltd, Ireland

\section{Brendan Bunting}

Ulster University, UK

\section{Edurne Garcia Iriarte}

Trinity College, Ireland

@SAGE

\begin{abstract}
A natural experiment contrasted the self-rated well-being of people with intellectual disabilities $(n=75)$ and those with enduring mental health problems $(n=44)$ after they moved to new accommodation and support options, while others remained in congregated settings or living in the family home. Most support staff also provided well-being ratings. In personalized arrangements, personal well-being was significantly higher than in congregated settings; particularly for people with intellectual disability who had higher support needs compared to people with mental health problems. Moving to a group home also brought some improvement in the well-being ratings of people with intellectual disability but only for those with higher support needs. Such moves seemed to lead to a decline in well-being for those with mental health problems. There were marked discrepancies between ratings given by the person with those of staff. The well-being measure shows promise for use in further comparative and longitudinal studies.
\end{abstract}

\section{Keywords}

intellectual disability, mental health problems, well-being, personalization, resettlement

\section{Corresponding author:}

Roy McConkey, University of Ulster, Jordanstown, Newtownabbey, BT37 0QB, UK.

Email: r.mcconkey@ulster.ac.uk 


\section{Introduction}

In most countries, the residential care of people with intellectual disabilities and of people with long-term mental health problems is still dominated by group-based accommodation and support (Mansell et al., 2010). Although the closure of large institutions is nearly complete in many affluent countries (Beadle-Brown et al., 2007), they have often been replaced by smaller scale arrangements in which groups of people live together; albeit with their own bedroom but sharing communal facilities and supported by staff employed by the service agency. However, a growing literature documents the aspirations of people with an intellectual disability to have their own home (Garcia Iriarte et al., 2014). The same aspirations are shared by people with enduring mental health difficulties as having a home facilitates their recovery (Shepherd et al., 2014). Thus, in recent years, there has been a shift away from shared living towards more individualized accommodation and support options, in which the person rents or owns a property and receives personalized support (Harkes et al., 2012). The arguments in favour of this approach are based mainly around human rights, better quality of life, increased self-determination and costeffectiveness (Fisher et al., 2007). Latterly, these developments have been spurred on by the expansion of new funding arrangements, such as individualized budgets in the United Kingdom, that permit support to be tailored to the needs of individuals and in some instances to enable people to employ their own support staff (Spicker, 2013).

Although government policy in the United Kingdom and Ireland is firmly supportive of personalized services (HM Government, 2009; Department of Health, 2012), the evidence remains sparse that such arrangements can deliver better outcomes for people with intellectual disability than the group living settings, which are still the dominant form of residential provision in many countries. It was against this background that the present evaluative study was conceived.

The Genio Trust was established in 2010 as an Irish registered charity with funding from Atlantic Philanthropies, the Health Services Executive and the Department of Health and Children. Providers of residential services for persons with disability or mental health problems could apply for grants from Genio to facilitate their service users in an individualized way, to move either from congregated settings to community alternatives or to more personalized arrangements in the community where they were living. An evaluation was then commissioned by Genio of the impact of these moves on people's lives (see McConkey et al., 2014 for full details). This article provides fuller analyses of changes in participants' subjective perceptions of his or her well-being.

Various measurement tools have been created based broadly around quality of life concepts and positive mental health (Townsend-White et al., 2012; Tennant et al., 2007). However, many of the existing scales do not take into account the specific challenges faced by persons with intellectual disabilities in providing self-assessments. The personal well-being index designed by Cummins and Lau (2005) is an exception as it was designed for persons with intellectual disability but using items that had been drawn from the wider literature on quality of life domains. Moreover, it had good psychometric properties and the potential to be used as a short, user-friendly monitoring tool by service personnel. Although it seemed suitable also for use with persons who had enduring mental health problems, this was tested through pilot studies and consultations with advisory and user groups. This process resulted in some modifications and the addition of further items to the well-being measure used in this evaluation.

This article compares the well-being ratings of people with intellectual disability and those with mental health problems who had moved from congregated settings to group homes with those who moved to personalized accommodation and support arrangements. The latter involved the person 
selecting and renting their accommodation and receiving an individualized support package provided by a service agency. Social security benefits covered their other expenditures. Over this same time period, some persons continued to live in congregated settings or with family carers as they awaited new support arrangements to be set up for them. In addition at the commencement of the evaluation, some people had already moved to personalized settings and remained there over the 2-year period from 2012 to 2014. Hence, there were five possible comparisons in what could be considered a natural experiment.

All of the participants in the study were drawn from a pool of persons who had been identified by their service providers for relocation and who would mostly continue to be served by these providers in the new arrangements. This reduced a possible confound in past research where the various types of accommodation and support arrangements were provided by different agencies to differently recruited groups of persons with intellectual disability. Indeed, this evaluation aimed to illustrate the impact on people's lives when service providers changed their model of residential supports. Moreover, this is one of the few studies that included people with enduring mental health problems both as a contrast with similarly aged persons with intellectual disability and also to explore the impact of living arrangements on their self-perceived well-being as this has been rarely researched (Priebe et al, 2009).

Finally, the study also provided an opportunity to contrast self-ratings of well-being with the ratings given by the support staff who had most involvement with the person. Proxy responding has often been used in past studies with persons who have intellectual disabilities and are used extensively in formal and informal evaluation of service provision. Greater awareness is needed about possible discrepancies between proxy and self-ratings; particularly in areas such as personal well-being (Emerson et al, 2013).

In summary, the aims of the study were:

- to monitor changes in the well-being ratings of people who move from congregated settings to group homes and personalized arrangements;

- to contrast the well-being ratings of people with intellectual disability and those with mental health problems and

- to compare ratings given by the person with those provided by their key workers.

\section{Method}

\section{Participants}

In all 18 service providers -6 mental health and 12 intellectual disability agencies - from across Ireland were grant aided by Genio Trust in 2010 and 2011, to facilitate the relocation of persons from congregated and group living settings to more personalized accommodation and support arrangements. A total of 119 persons were included in the study for whom personal well-being scores were available before and after moving: 75 of whom had an intellectual disability and 44 with mental health problems. Of these, $62 \%$ were male and $62 \%$ were aged 40 years of over. In addition, all but six were Irish and all but two were single.

Each person's support needs was assessed on 13 items related to daily life, such as, doing household chores, using public transport, shopping for groceries and filling in forms. A threepoint scale was used in the range of No support required, Some support, and Always needs support (Cronbach's $\alpha$ at time 1: 0.955 and time 2: 0.881). A count was then made of the number 
of items for which support was 'always needed' with high scores indicative of needing more support. Participants were then grouped into two levels of support needs depending on scores: lower support (0-8) and higher support (9-13). Only people with intellectual disability fell into the high support grouping.

At the start of the study, 29 persons had already moved to personalized arrangements and during it, 25 persons moved from congregated settings to personalized arrangements and 30 persons from congregated settings to group homes. In addition, a further 20 persons remained in congregated settings and 15 continued to live with family carers while awaiting a move to their own accommodation.

\section{Accommodation options}

Congregated settings. These included campus accommodation of separate bungalows and houses on a shared site as well as hospital wards. In such settings, people had little choice over whom they lived with; they shared communal bathing facilities, dining and sitting rooms and even though some had their own bedroom, others shared sleeping arrangements in ward-like accommodation. Staff were available $24 \mathrm{~h}$ a day although different staff might work across the various housing units and wards.

Personalized arrangements. Typically, these arrangements took the form of rented accommodation in ordinary houses or apartments with people either living alone $(n=45)$ or with a partner or friend of their choosing $(n=13)$. The tenants often chose the property and location as well as deciding on furnishings. Most had support staff visiting their home at agreed days and times for a set number of hours per week in accordance with their needs but a few individuals with higher support needs had staff available to them on a $24 / 7$ basis. Of the 29 people in the study who moved, 15 persons had lived in a group home, 9 in congregated settings and 5 with family carers. Twothirds of the persons in these settings reported that new staff were supporting them.

Group homes. These were ordinary housing in the community shared by up to six persons with support staff available during daytime hours or on a $24 \mathrm{~h}$ basis. However, these residents typically had a limited choice of co-residents. Prior to the move, 27 of these residents had lived in congregated settings and 3 in another group home. In all, $79 \%$ of the people reported that new staff were supporting them in their new home.

Family carers. A number of services were supporting people with intellectual disabilities who lived with family carers to move to their own accommodation with support. All had lived with families since childhood and were presently availing of a range of day support and short break services. One person lived with a foster family.

\section{Procedures}

Ethical approval was obtained from Ulster University (REC/11/0088). An easy-to-read information sheet describing the evaluation was prepared along with a consent form. These were sent to projects prior to the commencement of data gathering in the hope that they would be distributed to people supported, their relatives and key workers ahead of the interview. This did not always happen. Hence, all participants were talked through the information sheet and signed consent was obtained before the interview commenced.

In consenting, it was made clear to participants that they could refuse to answer any questions without giving a reason and they could terminate their participation at any time. They were assured 
that all their information would be kept private and no one, and no service would be identified in any reports. The support they received would be unaffected by their decision to participate or not.

A structured interview schedule was prepared in accordance with the wider aims of the evaluation. The questions in the schedule were informed by relevant literature, the specific aims of the projects supporting people to move and through consultation with an advisory group of service users. It was further revised after piloting. The well-being items were a discrete part of the schedule which also covered many other aspects of the person's living arrangements and daily activities.

A team of eight research associates was recruited and police clearance was obtained for them. They were trained to interview the persons with intellectual disability especially, the key worker of each participant and an available relative. The interviews were usually conducted in a private room in the person's residence. The person supported had the option of a member of staff or a relative being present. The interviews with them generally took around $2 \mathrm{~h}$ with breaks as needed but they could extend to nearly $4 \mathrm{~h}$.

The interviews took place on two occasions separated by an average of 24 months.

For the 119 interviews, $36 \%$ were undertaken with the person alone and the remainder $(64 \%)$ with the person and key worker. The interview data was transferred to SPSS version 22 for statistical analysis. Comparisons across the subgroups and the changes in accommodation and support options were made using $\chi^{2}$ tests or one-way analysis of variance as appropriate.

Well-being measure. The well-being measure had 10 items that covered health (physical and emotional), relationships, living arrangements, safety, respect and control (see Appendix Table 1A). A three-point rating scale was used - Not good, OK, and Great-accompanied by smiley faces for use with persons who had an intellectual disability to indicate their chosen response when the item was read out to them.

A total score was calculated across the 10 items (scores could range from 0 to 40 as ratings that were midway between two choices were counted on a scale $0,1,2,3$, and 4 ). The psychometric properties of the scale were determined as follows. A one-factor model within a confirmatory framework described the results for the 10 items, at the two points in time. The standardized factor loadings for the items ranged from 0.42 to 0.77 . The Cronbach's $\alpha$ for the well-being scale was 0.852 at time 1 and 0.833 at time 2 .

Also an 'improvement' score could be computed by subtracting well-being scores after the move with those before. A positive score indicated an improvement and a negative score a disimprovement. Overall, the individual improvement scores ranged from -20 to +30 .

The person's key workers were also asked to rate the person's well-being independently from the ratings given by the person or when they assisted the person. Their ratings were scored similarly. The Cronbach's $\alpha$ s for their ratings were 0.832 at time 1 and 0.775 at time 2 . However, these ratings were available for only $77 \%$ of participants as not all participants had access to a key worker.

\section{Results}

Table 1 presents the mean well-being scores given by persons before and after the moves. For comparison purposes, the mean scores of persons who remained in the same setting over the equivalent period of time are also included, namely, those who remained in congregated or family settings and those who continued to live in personalized arrangements. 
Table I. Means (and SDs) on well-being scores for the two groups at the two time points.

\begin{tabular}{llll}
\hline Living arrangements & Group & Time I & Time 2 \\
\hline Moved to group homes & Intellectual disability $(n=24)$ & $26.7(7.5)$ & $28.0(8.2)$ \\
& Mental health $(n=6)$ & $31.3(8.0)$ & $27.7(7.7)$ \\
Moved to personalized arrangements & Intellectual disability $(n=8)$ & $29.0(6.5)$ & $35.3(4.9)$ \\
& Mental health $(n=17)$ & $31.2(7.9)$ & $31.9(7.1)$ \\
Remained in congregated settings & Intellectual disability $(n=10)$ & $32.5(7.3)$ & $30.2(6.5)$ \\
& Mental health $(n=10)$ & $28.5(7.0)$ & $20.4(4.4)$ \\
Living in personalized arrangements & Intellectual disability $(n=19)$ & $32.7(6.4)$ & $35.2(4.2)$ \\
& Mental health $(n=10)$ & $29.2(7.5)$ & $27.8(7.6)$ \\
Lived with family carers & Intellectual disability $(n=15)$ & $32.1(11.0)$ & $36.0(5.5)$ \\
\hline
\end{tabular}

SD: standard deviation.

There were no significant differences in the mean scores across the five accommodation groupings at time 1 before the moves took place nor between ratings given by persons with intellectual disabilities and those by persons with mental health problems. Thus, the groups were matched on the main variable of interest. However, at time 2, after the moves had taken place, there were significant differences across the five groupings with people living in personalized settings or who had moved to them, having higher mean scores than those who continued to live in congregated settings $(F=6.6 ; p<0.001)$. Those living with family carers had the highest scores. Also people with intellectual disability gave significantly higher scores at time 2 compared to those with mental health difficulties (mean: 32.4; SD: 7.1 vs. mean: 27.6; SD: 7.9; $F=11.8 ; p<0.001$ ).

The changes in scores are further illustrated in Figure 1 which gives the mean improvement scores for the five subgroups. The largest improvements in well-being scores were rated by persons with intellectual disability who moved to personalized arrangements, but this was less marked for persons with mental health problems. Similarly, persons with intellectual disability who had previously moved to personalized settings also showed further improvements over the 2 years, whereas people with mental health problems had a mean disimprovement score over this time period. People with intellectual disability who continued to live with family carers also rated their personal well-being as having improved.

People with intellectual disability who moved to group homes showed a slight mean improvement in their well-being ratings but people with mental health problems generally had lower scores, although the number of persons in this grouping was small $(n=6)$. However, the biggest drop in well-being scores was for those who continued to reside in congregated settings and particularly those with mental health problems.

\section{Support needs}

The improvement ratings given by people with intellectual disability were further investigated in terms of their needs for higher or lower support. (Note: all persons with mental health problems had low support needs.) Due to the small numbers, people already living in personalized arrangements and those who moved to them were combined into one grouping. As Figure 2 illustrates, people in these settings had improved well-being scores but the mean scores were significantly higher for persons with higher support needs. For people moving to group homes, those with higher support needs had higher mean improvement scores, whereas the scores of persons with lower support 


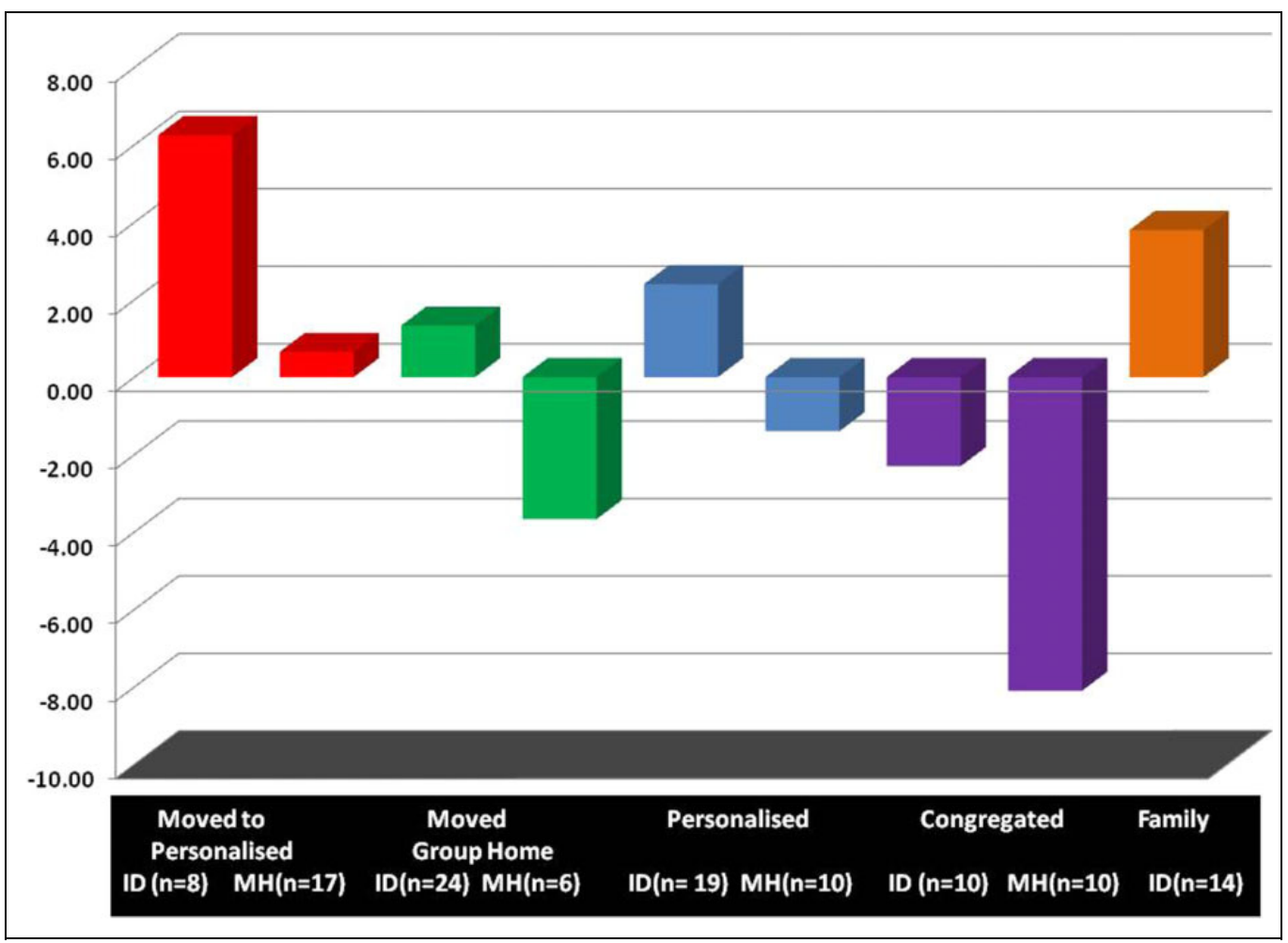

Figure I. Mean improvement scores on well-being measure for the five subgroups.

needs had disimproved. Those who continued to live in congregated settings tended to have lower mean scores irrespective of their support needs. People living with family carers who had higher support needs also had higher improved scores compared to those with lower support needs.

Predictors of well-being. In order to examine the interrelationships among the possible predictors of well-being, the total scores at time 2 were regressed onto the equivalent measure at time 1 with three covariates introduced to the structural equation model (analysis of covariance model). These were the type of living arrangements, intellectual disability or mental health problems, and the level of support required by the person. This model was a good description of the data $\left(\chi^{2}=288.77 ; d f=246 ; \mathrm{pr}=0.03\right.$; root mean square error of approximation $=0.035 ; 90 \%$ confidence interval $=0.011,0.050$; comparative fit index $=0.947$; standardized root mean residuals $=0.08$ ).

The covariates affected the well-being scores on both occasions. At time 1, those participants with an intellectual disability $(\mathrm{Est} / \mathrm{SE})=1.76 ; p<0.07)$ and/or who required low levels of support (Est/SE $=3.02 ; p<0.01)$ tended to have higher scores. However, at time 2, the level of support was no longer significant, which suggests that persons with higher support needs had well-being scores equivalent to persons with low support needs but living in personalized arrangements (Est/SE = $3.21 ; p<0.001)$ and having an intellectual disability (Est/SE $=4.49 ; p<0.001)$ continued to be highly significant. 


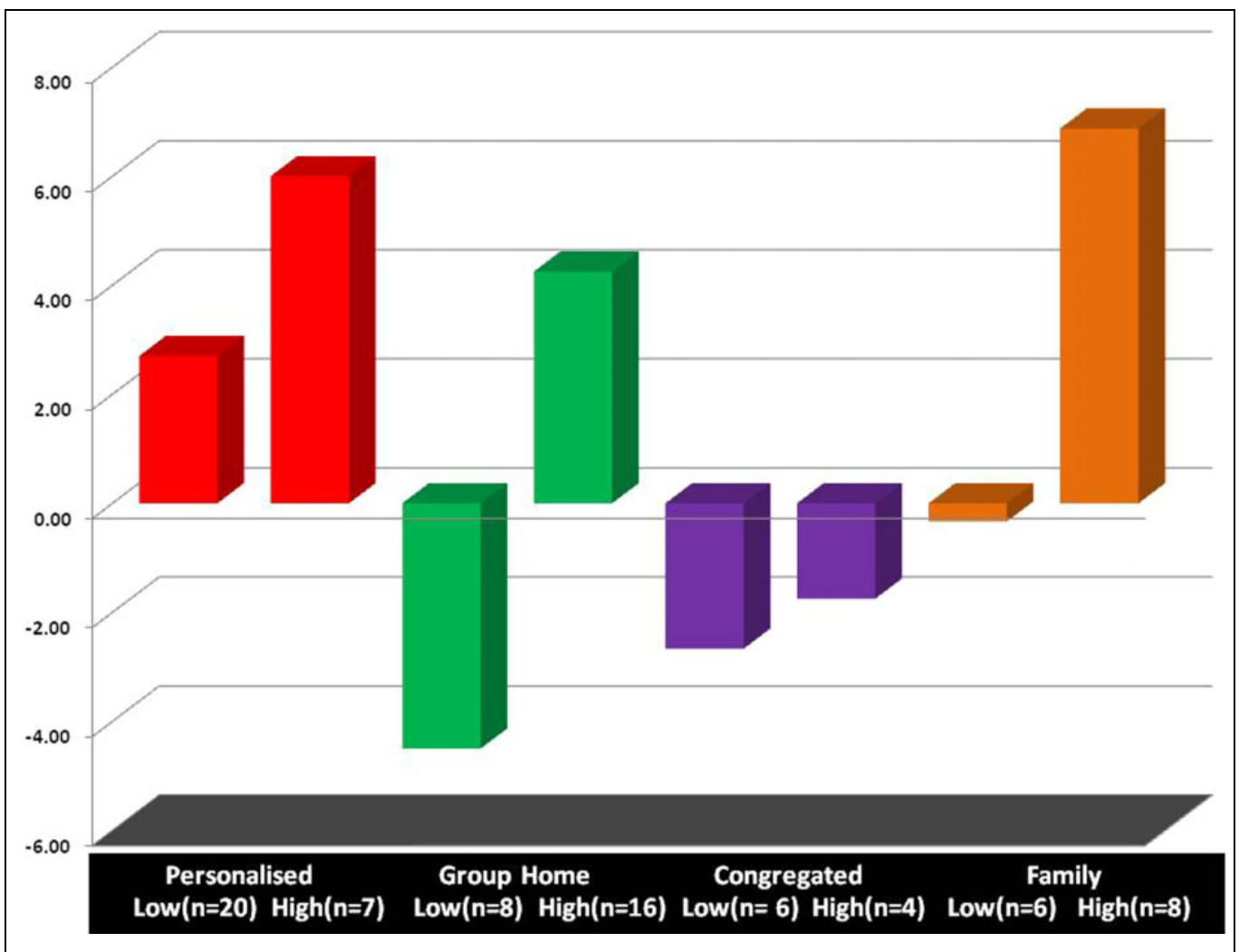

Figure 2. Mean improvement scores on well-being measure for persons with intellectual disability by support needs in four accommodation options.

\section{Staff ratings}

The correlations between the well-being scores given by the person and by their key workers based on their knowledge of the person were $r=0.551(n=93)$ at time 1 and $r=0.329(n=93)$ at time 2. (The latter information was missing for 26 persons as key workers were either not available or they declined to provide the information.) The correlation between the two informant groupings on the improvement scores was $r=0.215$ which, although statistically significant, indicates a low proportion of shared variance.

Figure 3 illustrates the mean improvement scores given by key workers for the five subgroups. Their highest improvement scores were for people who moved to group homes with disimprovements recorded for persons living with family carers $(F=2.60 ; p<0.05)$. However, comparison of Figure 3 with Figure 1 indicates that people's own improvement ratings were much lower. Indeed, staff consistently rated the well-being of persons with mental health problems overall as improved, whereas the people themselves rated it much lower (person mean: -1.19 : key worker mean: $3.5 ; t=-2.88 ; p<0.01$ ). By contrast, people with intellectual disability tended to rate their well-being higher than did staff at time 1 especially (person mean score at time 1: 30.45 ; key worker mean score at time $2: 27.67 ; t=3.5 ; p<0.001$ ). However, the only statistically significant difference between the mean improvement scores given by staff compared to that of 


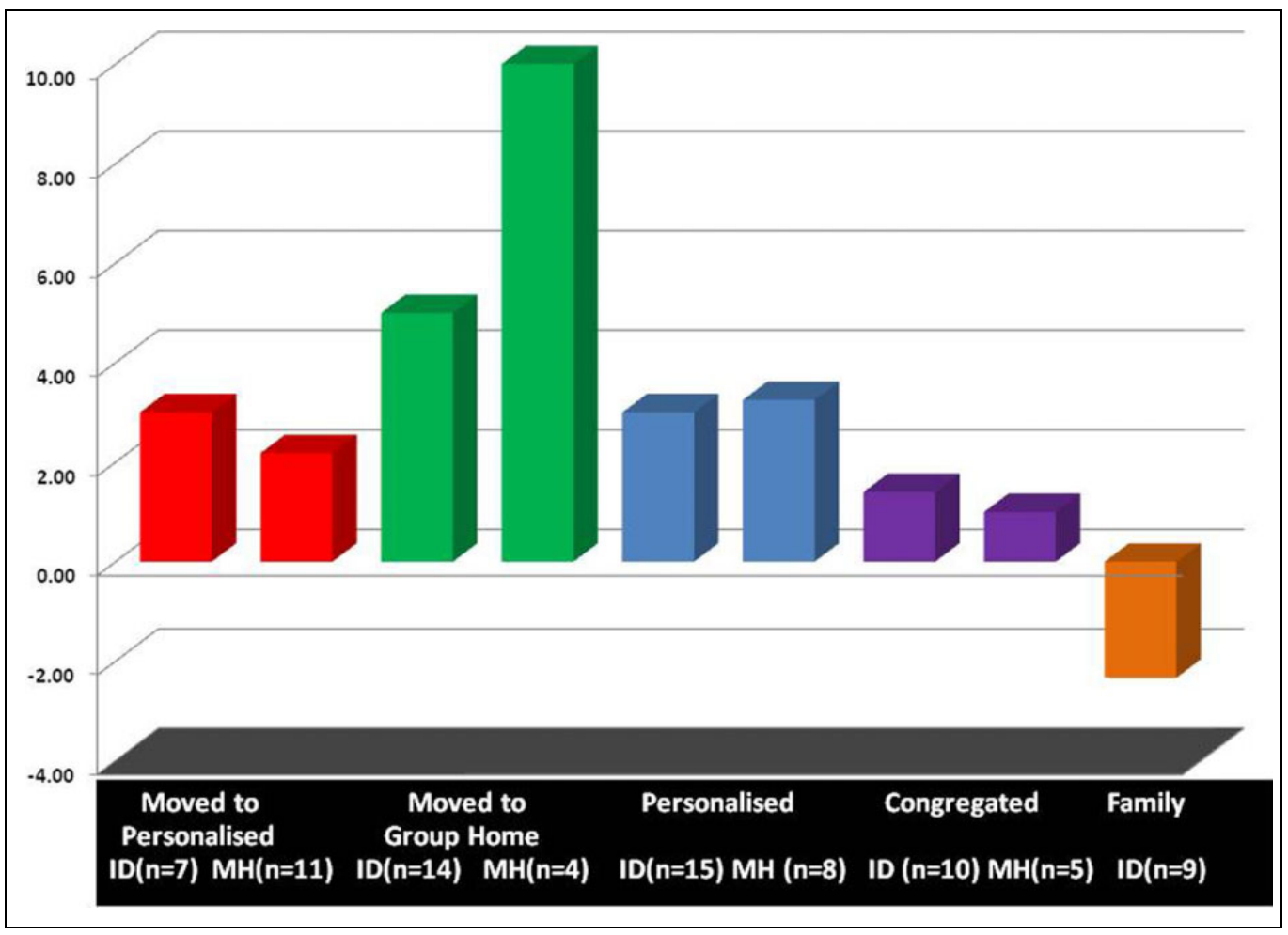

Figure 3. Mean improvement scores on the well-being measure given by key workers for the five subgroups.

people they supported was in congregated settings $(t=12.83 ; p<0.01)$ although the group home ratings just failed to be significant $(p<0.06)$.

\section{Discussion}

This study is limited in a number of respects. It included a self-selected sample of services and persons who choose to make changes in their living arrangements. They are unlikely to be representative of all persons living in these different settings. Also the small number of persons within certain subgroupings is a further limitation. Moreover, it was not possible to match the persons on other characteristics across the groupings that might have further influenced their well-being and could account for the variation in scores within each of the subgroupings, such as their mental health diagnosis.

That said, the main value of this natural experiment is that the same persons rated their wellbeing at two time points and the inclusion of two comparator groups, namely, persons with intellectual disability and those with enduring mental health problems. During the 2-year period, some people had moved to new accommodation, whereas others remained in the same setting. There is a strong evidence that those persons living in, or who moved to personalized arrangements, rated their personal well-being significantly higher than those who remained in congregated settings. This applied more so to the people with intellectual disability 
who moved - particularly those with higher support needs -than it did for people with mental health problems. Indeed, for the latter group, there was the possibility of a decrease in their well-being over the 24 month period.

Moving to a group home also brought some improvement in the personal well-being of people with intellectual disability but only for those with higher support needs, whereas there was the suggestion that such moves led to a disimprovement in well-being for those with mental health problems. Group living arrangements may continue some of the practices associated with congregated settings such as residing with other people whom you have not chosen; the supervisory role of staff and limited opportunities to make decisions; all of which limits the autonomy of the residents (McConkey et al., 2016).

By contrast, personalized accommodation and support arrangements enhanced the well-being of people with intellectual disability and particularly those with higher support needs. This indicative finding requires further exploration but may relate to the increased opportunities given to such persons around self-determination, choice, privacy and community engagement which they had been denied in previous residential arrangements and family care (Curryer et al., 2015; Lachapelle et al., 2005; Ticha et al., 2012).

By contrast, there is the suggestion that people with mental health problems fare less well in personalized settings as time goes on. Given the small numbers of persons in this study, this finding must be treated with particular caution and requires further explication through longitudinal studies and larger samples. Of particular interest could be the reduced support available to persons living alone, especially if there is deterioration in their mental health (Leff et al., 2009; Priebe et al., 2009). Alternatively, this finding could be due to the stress and upheaval of moving to new surroundings and may reduce over a longer time period as people settle in.

Of particular note is the discrepancy between well-being ratings given by the person and those based on the perceptions of their support staff. Overall, the correlations were low - albeit statistically significant - with the discrepancy particularly marked for persons with mental health problems and with those living in congregated and group settings. Staff may base their ratings on the physical attributes of the settings in which people live, whereas the persons draw on their feelings and experiences. It is a salutary reminder that proxy responses around personal well-being can be misleading for people who may have limited communication and the importance of striving to find methods for ensuring their opinions are sought (Emerson et al., 2013).

People with intellectual disability who lived with family carers tended to score highest in terms of their personal well-being and yet all had requested a move from the family home to having their own place. Thus, personal aspirations need to be taken into account when it comes to decisions around where people live and with whom they live, even though there may be a risk that their personal well-being could reduce, at least as measured by this scale (Curryer et al., 2015; Scott et al., 2014).

This is one of the few longitudinal studies of the impact which changes in accommodation and support arrangements can have on the well-being of persons with intellectual disabilities and on those with enduring mental health problems. Nevertheless, further research could usefully monitor changes in personal well-being over longer periods of time and the tool used in this study shows promise as a simple, user-friendly tool which service personnel can use to do this with the people they support. However, such evaluations need to be set within a review process of person-centred planning so that areas in which well-being is weak can be addressed. This process would also provide insights into the variability in personal well-being among people living in similar accommodation options that this and other studies have reported. 


\section{Acknowledgements}

We thank the project manager Rachel Stevens and our research assistants: Mairead Brennan, Noelle Fitzgerard, Anna Giblin, Gina McNamara, Sheelah Flatman-Watson, Siobhan O'Doherty and Maeve O'Halloran. We also thank Sharon Brady for her help with data coding.

\section{Declaration of Conflicting Interests}

The Director of Research and Evidence for Genio Trust is one of the authors (FK). The author(s) declared no potential conflicts of interest with respect to the research, authorship, and/or publication of this article.

\section{Funding}

The author(s) disclosed receipt of the following financial support for the research, authorship, and/or publication of this article. This evaluation was commissioned and funded by Genio Trust.

\section{References}

Beadle-Brown J, Mansell J and Kozma A (2007) Deinstitutionalization in intellectual disabilities. Current Opinion in Psychiatry 20: 437-442.

Cummins RA and Lau ALD (2005) Personal Wellbeing Index - Intellectual Disability Manual, 3rd ed. Hong Kong: Polytechnic University.

Curryer B, Stancliffe RJ and Dew A (2015) Self determination: adults with intellectual disability and their family. Journal of Intellectual and Developmental Disability 40: 394-399.

Department of Health (2012) Value for Money and Policy Review of Disability Services in Ireland. Dublin: Department of Health.

Emerson E, Felce D and Stancliffe RJ (2013) Issues concerning self-report data and population-based data sets involving people with intellectual disabilities. Intellectual and Developmental Disabilities 51(5): 333-348.

Fisher KR, Parker S, Purcal C, et al. (2007) Effectiveness of Supported Living in Relation to Shared Accommodation, Report prepared for the Disability Policy and Research Working Group. Sydney: University of New South Wales.

Garcia Iriarte E, O'Brien P, McConkey R, et al. (2014) Identifying the key concerns of Irish persons with intellectual disabilities. Journal of Applied Research in Intellectual Disabilities 27(6): 564-575.

Harkes MA, Brown M and Horsburgh D (2012) Self directed support and people with learning disabilities: a review of the published research evidence. British Journal of Learning Disabilities 42: 87-101.

HM Government (2009) Valuing People Now: A New Three-year Strategy for People with Learning Disabilities. London: Department of Health.

Leff S, Chow CM, Renee Pepin MA, et al. (2009) One size fit all? What we can and can't learn from a meta-analysis of housing models for persons with mental illness. Psychiatric Services 60 (4): 473-482.

Lachapelle ML, Wehmeyer MC, Haelewyck Y, et al. (2005) The relationship between quality of life and self-determination: an international study. Journal of Intellectual Disability Research 49(10): 740-744.

McConkey R, Bunting B, Ferry F, et al. (2014) An Evaluation of Personalised Supports to Individuals with Disabilities and Mental Health Difficulties. Mullingar: Genio Trust.

McConkey R, Keogh F, Bunting B, et al. (2016) Relocating people with intellectual disability to new accommodation and support settings: contrasts between personalised arrangements and group home placements. Journal of Intellectual Disabilities 20(2): 109-120.

Mansell J and Beadle-Brown J; Members of the Special Interest Research Group on Comparative Policy and Practice (2010) Deinstitutionalisation and community living: position statement of the Comparative Policy and Practice Special Interest Research Group of the International Association for the Scientific Study of Intellectual Disabilities. Journal of Intellectual Disability Research 54(2): 104-112. 
Priebe S, Saidi M, Want A, et al. (2009) Housing services for people with mental disorders in England: patient characteristics, care provision and costs. Social Psychiatry and Psychiatric Epidemiology 44: 805-814.

Shepherd G, Boardman J, Rinaldi M, et al. (2014) Supporting recovery in mental health services: quality and outcomes. London: Centre for Mental Health and Mental Health Network, NHS Confederation 2014

Spicker P (2013) Personalisation falls short. British Journal of Social Work 43: 1259-1275.

Tennant R, Hiller L, Fishwick R, et al. (2007) The Warwick-Edinburgh mental well-being scale (WEMWBS): development and UK validation. Health and Quality of Life Outcomes 5 (63): 1-13.

Ticha R, Lakin C, Larson SA, et al. (2012) Correlates of everyday choice and support-related choice for 8,892 randomly sampled adults with intellectual and developmental disabilities in 19 states. Intellectual and Developmental Disabilities 50(6): 486-504.

Townsend-White C, Pham ANT and Vassos MV (2012) A systematic review of quality of life measures for people with intellectual disabilities and challenging behaviours. Journal of Intellectual Disability Research 56(3): 270-284.

Scott M, Foley K-R, Bourke J, et al. (2014) "I have a good life": the meaning of well-being from the perspective of young adults with down syndrome, Disability and Rehabilitation 36(15): 1290-1298.

\section{Appendix I}

Table IA. Well-being indicators.

\begin{tabular}{|c|c|c|c|c|}
\hline Overall how is... & 1 & 2 & 3 & DK \\
\hline \multicolumn{5}{|l|}{ Practice item: the food you get in the place where you live } \\
\hline \multicolumn{5}{|l|}{ Life for you at present } \\
\hline \multicolumn{5}{|l|}{ Your physical health (probe: keeping well, not being sick) } \\
\hline \multicolumn{5}{|l|}{ Your mental well-being (probe: feeling good inside, happy, relaxed) } \\
\hline \multicolumn{5}{|l|}{ Your feeling safe when you go out (probe: no one bullies or harms you) } \\
\hline \multicolumn{5}{|l|}{$\begin{array}{l}\text { Your relationships with your family (probe: getting on with your family } \\
\text { and doing things with them.) }\end{array}$} \\
\hline \multicolumn{5}{|l|}{$\begin{array}{l}\text { Your relationships with your friends (probe: seeing them more often } \\
\text { and getting along with them) }\end{array}$} \\
\hline \multicolumn{5}{|l|}{$\begin{array}{l}\text { Your relationship with support staff (probe: having people to help you } \\
\text { to do the things that you want to do) }\end{array}$} \\
\hline \multicolumn{5}{|l|}{ The place where you live (probe: the facilities, the neighbourhood) } \\
\hline \multicolumn{5}{|l|}{$\begin{array}{l}\text { The respect you get from the people who support you (probe: how they } \\
\text { speak to you; asking your opinion, listening to you). }\end{array}$} \\
\hline $\begin{array}{l}\text { The control you have in your life (probe: making decisions for yourself } \\
\text { rather than other people doing it for you) }\end{array}$ & & & & \\
\hline
\end{tabular}

Note: How is life for you at present? Is it good or not so good? I am going to ask some questions. For each question you have three choices. I - Not good; 2 -OK; 3 - It's Great (show three cards with smiley faces). Point to the one that describes best how you feel. (If person is unsure or does not know, tick Don't know (DK) column.) 